\title{
Isolation and Screening of Microbial Isolates from Kombucha Culture for Bacterial Cellulose Production in Sugarcane Molasses Medium
}

\section{Clara Angela, Jeffrey Young, Sisilia Kordayanti, Putu Virgina Partha Devanthi, and Katherine}

Department of BioTechnology, School of Life Sciences, Indonesia International Institute for Life Sciences, Jalan Pulomas Barat Kav. 88, Kayu Putih, Pulo Gadung, Jakarta Timur

\section{Abstract}

Kombucha tea is a traditional fermented beverage of Manchurian origins which is made of sugar and tea. The fermentation involves the application of a symbiotic consortium of bacteria and yeast (SCOBY) in which their metabolites provide health benefits for the consumer and subsequently allow the product to protect itself from contamination. Additionally, kombucha tea fermentation also produces a byproduct in the form of a

Corresponding Author:

Katherine

katherine.k@i3ı.ac.id

Received: 1 February 2020

Accepted: 8 February 2020

Published: 16 February 2020

Publishing services provided by Knowledge E

(c) Clara Angela et al. This article is distributed under the terms of the Creative Commons

Attribution License, which permits unrestricted use and redistribution provided that the original author and source are credited.

Selection and Peer-review unde the responsibility of the IC-BIOLIS Conference Committee. pellicle composed of cellulose (Bacterial Cellulose, BC). Compared to plant cellulose, BC properties are more superior, which makes it industrially important. However, BC production at industrial scale has been faced with many challenges, including low yield and high fermentation medium cost. Many researchers have focused their studies on the use of alternative low cost media, such as molasses, which is a by-product of sugar refining process. To maximize the BC production in molasses medium, it is important to select the microbial strains that can grow and produce $B C$ at high yield in molasses. This study aimed to isolate and characterize BC-producing bacteria and a dominant yeast from kombucha culture which had been previously adapted in molasses medium. The isolation of bacteria was performed using Nutrient Agar (NA) and Hestrin and Schramm (HS) supplemented with cycloheximide, while yeast was isolated using Potato Dextrose Agar (PDA) supplemented with chloramphenicol. The most dominant colonies were isolated and then subjected to microscopic observation for morphological analysis. The pure bacteria and yeast isolates were then identified by sequencing the 16S rRNA gene and D1/D2 region of the 26S rRNA, respectively. The bacteria isolates obtained were all from closely related genera: Komagataeibacter sp. DS1MA.62A, Komagataeibacter xylinus, Komagataeibacter saccharivorans, Komagataeibacter xylinus and Gluconacetobacter saccharivorans. The single isolated yeast was identified as Brettanomyces bruxellensis. This study helps to elucidate the BC-producing species which thrive in molasses medium for potential use in the $B C$ production using molasses as alternative cheap carbon source. Also, the study revealed that the co-culture of Komagataeibacter sp. DS1MA.62A and B. bruxellensis could produce BC from molasses supplemented with caffeine and acetate buffer at an average yield of $27.7 \pm 1.83 \mathrm{~g} / \mathrm{L}$.

Keywords: Kombucha, SCOBY, bacterial cellulose, Acetobacter, Komagataeibacter, Brettanomyces, alternative medium, molasses, caffeine, acetate buffer. 


\section{Introduction}

Kombucha tea is a traditional fermented beverage originated from northeast China, Manchuria [1]. Nowadays, it has become very popular in Asia and Western world (North America and Europe) due to many health claims that are linked to its chemical composition resulted from microorganisms activity during fermentation process. The tea is traditionally made by fermenting sugar-rich black or green tea with indigenous microorganisms mostly known as SCOBY, a short for symbiotic consortium of bacteria and yeast [1, 2]. The main product coming from the fermentation is a slightly acidic or sour beverage known as kombucha tea. It is rich in organic acids such as acetic, gluconic, glucuronic, citric, L-lactic; and vitamins B1, B2, B6, B12, C, as well as 14 amino acids [1]. The fermentation not only produces the tea, but it also by-produces a transparent pellicle of cellulose called bacterial cellulose or BC.

$\mathrm{BC}$ has recently gained a huge interest due to better physical and chemical properties compared to plant cellulose such as higher purity, crystallinity, polymerization, tensile strength, and water holding capacity, although both have the same molecular formula $\left(\mathrm{C}_{6} \mathrm{H}_{10} \mathrm{O}_{5}\right)_{n}$ [3--5]. Those superiority of $\mathrm{BC}$ leads to huge potential in industry, replacing cellulose derived from plant. Recent research has revealed the $\mathrm{BC}$ potential as biosorbent in waste management industry, thickening agent and stabilizer in the food industry, and wound healing agent in pharmacy and medical field $[1,3,5]$.

Despite the enormous potential of $\mathrm{BC}$, its industrial production is still hindered by high fermentation medium cost. Industrial scale fermentation generally utilizes pure sugars such as glucose, sucrose, mannitol, fructose, and arabitol as carbon source, which consume about $30 \%$ of the total production cost [6]. Recent studies have revealed the potential of carbon-rich agroindustrial waste such as molasses, wheat straw, rice bark, and many more as an alternative fermentation medium for producing BC [6--8]. The strategy of utilizing waste as alternative medium could not only lower the production cost, but also solve the environmental issues that might arise from unprocessed waste.

Fermentation process to produce kombucha tea beverage along with $\mathrm{BC}$ involves bacteria and yeast which exist as a symbiotic consortium (SCOBY). In the case where the fermentation uses complex sugar, the yeast helps the bacteria to digest the complex sugar sucrose into glucose and fructose. The sugar digestion by yeast will also produce ethanol through hydrolysis and glycolysis pathway [1,9]. The glucose and ethanol derived from yeast metabolism are utilized as a food source by acetic acid bacteria where they oxidize them into gluconic acid and acetic acid [1,9]. The metabolism process can be seen in Figure 1. Symbiotic relation between S. cerevisiae and Acetobacter 
isolated from Haipao fermentation was reported by Liu et al. (1996). They mentioned that the yeast produced ethanol to drive the bacterial growth and thus proportionally promoting higher acetic acid production. The symbiotic relationship happened in a way that ethanol production by yeast can be further stimulated by acetic acid [10--12]. The presence of ethanol has also been confirmed by several previous studies to positively affect the BC yield [11, 12].

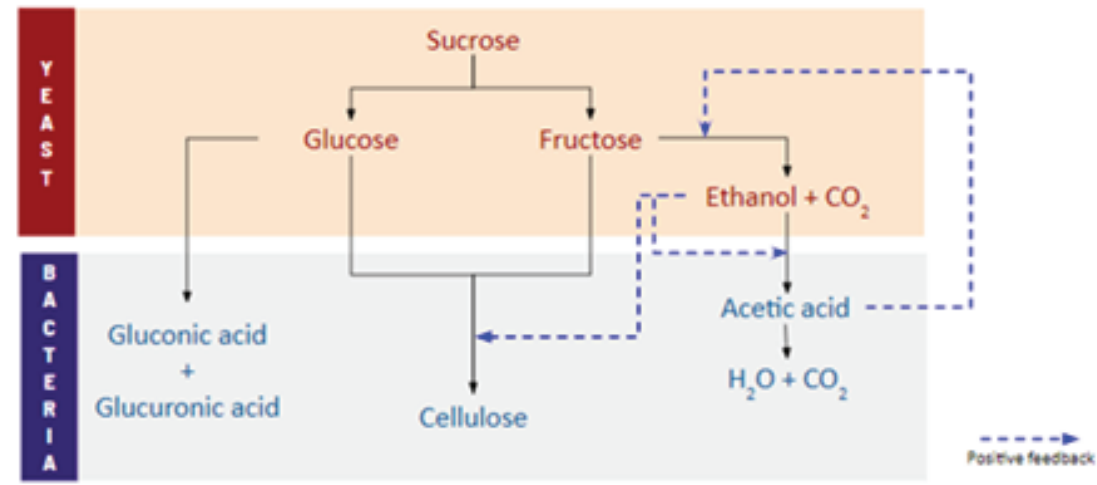

Figure 1: Microbial Interaction in Kombucha Tea Fermentation. (Villarreal-Soto et al., 2018, after modification)

Microbial composition of SCOBY may vary, depending on the inoculum source as well as fermentation condition; however, it is generally composed of acetic acid bacteria (AAB), lactic acid bacteria (LAB), and yeast [2, 13]. Several previous studies have successfully identified the most dominant bacteria and yeast species. The bacteria is generally coming from the genus Gluconacetobacter, Agrobacterium, Aerobacter, Alcaligenes, Achromobacter, Azotobacter, Sarcina, Rhizobium, Salmonella, and Escherichia [3--5]. The most well-known and well-studied cellulose-producing bacteria is Komagataeibacter xylinus, which was formerly known as Acetobacter xylinum. Other cellulose-producing bacteria that have also been identified are Komagataeibacter kombuchae, Gluconacetobacter sp A4, Gluconacetobacter sacchari, Acetobacter aceti, Acetobacter nitrogenificans, Acetobacter pasteurianus, Acetobacter liquefaciens, Bacterium gluconicum, and Gluconobacter oxydans $[1,14]$. On the other hand, the yeast ecology in SCOBY is dominated by Saccharomyces, Saccharomycodes, Zygosaccharomyces, Candida, Pichia, Schizosaccharomyces, Brettanomyces/Dekkera, Torulospora, Koleckera, Mycotorula, and Mycoderma [1, 9, 15, 16]. All of them are described as osmotolerant, acid-tolerant, fermentative, and alcohol- as well as flavor-producing yeast.

In addition to suitable fermentation medium, the use of appropriate microbial strain also plays a key role in enhancing the $\mathrm{BC}$ production. To maximize the $\mathrm{BC}$ production in molasses, the microbial strains used should be able to grow and exhibit a high BC production capacity in molasses. Since molasses consists primarily of sucrose 
(50\%), the utilization of yeast could help break down the sucrose into fructose and glucose, which will subsequently be used by the bacteria to synthesize BC. In this study, kombucha cultured suspected to contain yeast and BC-producing bacteria was adapted in molasses. The predominant bacteria and yeast were then isolated and identified by sequencing the 16S rRNA gene and D1/D2 region of the 26S rRNA gene, respectively. $\mathrm{BC}$ production from molasses with co-culture consisting the dominant bacterial and yeast isolates was also addressed in this study.

\section{Methods}

\subsection{Materials}

Kombucha tea was kindly provided by PT Tujju Kombucha (Jakarta, Indonesia). Goalpara Perbawati black tea (Sukabumi, Indonesia) and Gulaku Murni table sugar from Sugar Group Companies (Lampung, Indonesia) were purchased from a local market in Indonesia and were used in the starter culture preparation step. Molasses that was used as the carbon source in the study was supplied by PT. Andalan Furnindo (Marunda, Indonesia). The characteristics of the molasses presented in Table $\mathbf{1}$ was provided as well by the company. Pure caffeine with minimal purity of $99.5 \%$ used in the study was purchased from local online shop. Other chemicals used in this study were purchased from Sigma-Aldrich and Merck.

TABLE 1: Characteristics of Molasses Used in the Current Study.

Parameter
Color
Brix
Sucrose
Inverted sugar
Purity
Specific gravity
pH
Ash Conductivity

\begin{tabular}{|c|}
\hline Unit \\
\hline visual \\
\hline$\%$ \\
\hline$\%$ \\
$\%$ \\
$\%$ \\
$\%$ \\
\hline
\end{tabular}

\begin{tabular}{|c|}
\hline Result \\
\hline Brown/Black \\
77.6 \\
46.92 \\
60.76 \\
60.76 \\
1.39835 \\
5.12 \\
0.4
\end{tabular}




\subsection{Kombucha culture adaptation in molasses}

This step was performed in order to adapt the culture present in the kombucha tea to molasses medium. The adaptation process was done sequentially as described in Figure 2. The first adaptation was done in sugared tea medium that was prepared by boiling the black tea and table sugar at concentration of $100 \mathrm{~g} / \mathrm{L}$ and $10 \mathrm{~g} / \mathrm{L}$, respectively, in type III water for 10 minutes. The tea mixture was then filtered through filter paper $125 \mathrm{~mm} \varnothing$ (cat no 1004125 Whatman) in a funnel into $100 \mathrm{ml}$ Erlenmeyer flask and autoclaved at $121^{\circ} \mathrm{C}$ for 20 minutes. After being autoclaved, the mixture was left to cool down and inoculated with kombucha tea; the size of the inoculum was $10 \%$ of the final volume. The setup was then left in a dark room at room temperature $\left(28 \pm 2{ }^{\circ} \mathrm{C}\right)$ for 7 days to be used in the second step of adaptation. The fermentation medium used in the second adaptation step was prepared by mixing molasses at $50 \mathrm{~g} / \mathrm{L}$ and filtered sugared tea that was prepared by boiling tea at $10 \mathrm{~g} / \mathrm{L}$ and table sugar at $50 \mathrm{~g} / \mathrm{L}$. The mixture was then autoclaved at $121{ }^{\circ} \mathrm{C}$ for 20 minutes then left to cool down before being inoculated with culture from step 1 . The set up was left in a dark room at room temperature for 5 days. The last adaptation part was done by inoculating culture from step 2 in sterilized molasses medium that was prepared by dissolving $100 \mathrm{~g} / \mathrm{L}$ molasses in acetate buffer $(200 \mathrm{mM}, \mathrm{pH}$ 4.75). The set up was left in a dark room for 6 days. The composition of fermentation in each step can be seen in Table 2.

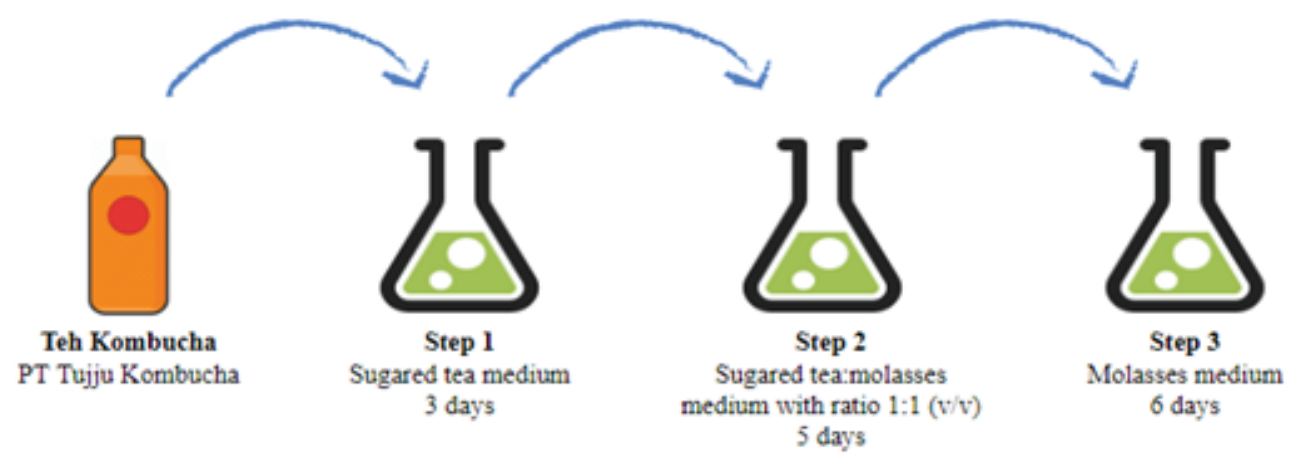

Figure 2: Schematic Procedure for Starter Culture Preparation. The composition of fermentation medium in each step can be seen in Table 2.

\subsection{Isolation and Characterization of Microbes in Molasses-Adapted Kombucha Culture}


TABLE 2: Composition of Medium Used for Adaptation.

\begin{tabular}{l|c|c|c|} 
& Step 1 & Step 2 & Step 3 \\
\hline Black tea (g/L) & 10 & 10 & - \\
\hline Table sugar (g/L) & 100 & 50 & 150 \\
\hline Molasses (g/L) & - & 50 & 1000 \\
\hline Total Volume (ml) & 50 & 200 & - \\
\hline Water (ml) & 45 & 180 & 900 \\
\hline 200mM acetate buffer pH $4.75(\mathrm{ml})$ & - & - & $100^{c}$ \\
\hline Culture $(\mathrm{ml})$ & $5^{a}$ & $20^{b}$ & \\
\hline
\end{tabular}

${ }^{a}$ Kombucha tea, ${ }^{b}$ Fermentation broth from step $1,{ }^{c}$ Fermentation broth from step 2

\subsubsection{Microbiological Culture Media Preparation}

Nutrient Agar (NA) and Potato Dextrose Agar (PDA) were obtained from Sigma-Aldrich. Both NA and PDA were prepared according to the manufacturer's instructions. HestrinSchramm (HS) agar was made by mixing $20 \mathrm{~g} / \mathrm{L}$ of glucose, $5 \mathrm{~g} / \mathrm{L}$ of peptone, $5 \mathrm{~g} / \mathrm{L}$ of yeast extract, $2.7 \mathrm{~g} / \mathrm{L}$ of $\mathrm{Na}_{2} \mathrm{HPO}_{4}$, and $1.15 \mathrm{~g} / \mathrm{L}$ of citric acid. To prepare plates for enumeration of bacteria from kombucha fermentations, a $0.5 \mathrm{mg} / \mathrm{mL}$ solution of cycloheximide in ethanol was added to cooled, molten NA and HS. To prepare plates for enumeration of yeasts from kombucha fermentations, a $25 \mathrm{mg} / \mathrm{mL}$ solution of chloramphenicol in ethanol was added to cooled, molten PDA.

\subsection{Isolation of Dominant Microbes (Bacteria and Yeast) in Kom- bucha Culture Adapted in Molasses}

Fermentation broth from step 3 in adaptation part was cultured on 3 types of agar: PDA, $\mathrm{NA}$, and HS. The sample (10 ml fermentation broth) was first serially diluted in sterile phosphate buffer saline (PBS). The bacteria was isolated on NA media that had been added with cycloheximide to inhibit the growth of yeast, which was then incubated at room temperature for 24-48 hours. On the other hand, the yeast was isolated on PDA which had been added with chloramphenicol to inhibit the growth of bacteria, followed by incubation at room temperature for 72 hours. Microbial samples were also grown on HS agar media at room temperature for 72 hours. The dominant isolates of each petri dish were selected based on colony morphology and the result of gram staining. 


\subsection{Characterization and Identification of Bacteria and Yeast Iso- lates}

Bacterial isolates were identified through PCR method and gene sequencing of the 16S rRNA gene using universal primers (27F/1492R). Meanwhile yeast isolates were identified through PCR method and gene sequencing of the D1/D2 region of the $26 \mathrm{~S}$ rRNA gene using primers NL-1 and NL-4. The sequence of the primers are listed in Table 3. PCR product was then run on electrophoresis in $1 \%$ agarose gel with Tris-borate-EDTA. Agarose gel was then soaked in ethidium bromide and visualized with the help of UV light. Then, the pure PCR results from bacterial 16S rRNA gene amplification and yeast 26S rRNA were sent for sequencing.

\subsection{Evaluation of the Potential of Bacteria Isolates for Producing BC Pellicle}

In this step, bacterial isolates were tested exclusively, as yeast are not celluloseproducing microbes. Bacteria isolates obtained from previous step were tested for their potential to produce $\mathrm{BC}$ by growing each isolate on a fermentation medium containing $150 \mathrm{~g} / \mathrm{L}$ molasses and $500 \mathrm{mg} / \mathrm{L}$ pure caffeine dissolved in acetate buffer solution (200 $\mathrm{mM}, \mathrm{pH}$ 4.75). This stage was carried out on 6-well plates and the fermentation was done at room temperature for 7 days. The isolate with the highest yield (significant BC yield expressed in $\mathrm{g} / \mathrm{L}$ ), was selected to produce $\mathrm{BC}$ in the next step.

\subsection{BC Pellicle Production through Fermentation of Molasses with Co-culture of Isolated Microbes}

According to the results obtained from previous steps, the most productive bacteria (Komagataeibacter sp. DS1MA.62A) was selected to be used for the following experiment. In this stage, BC was produced through fermentation with co-culture consisted of the bacteria and yeast isolate (Brettanomyces bruxellensis). The fermentation medium consisted of $500 \mathrm{mg} / \mathrm{L}$ caffeine and $150 \mathrm{~g} / \mathrm{L}$ molasses prepared in acetate buffer (200 mM, pH 4.75). Fermentation volume was $3 \mathrm{~mL}$, using 6 -well plates. The medium was autoclaved first before being inoculated with co-culture of bacteria and yeast. The fermentation was carried out at room temperature for 14 days. After 14 days of fermentation, the $\mathrm{BC}$ pellicle was harvested and dried at $50^{\circ} \mathrm{C}$ in an oven until the weight remained constant. The dry weight of $\mathrm{BC}$ pellicle was recorded and reported in this study as BC yield expressed in $\mathrm{g} / \mathrm{L}$, which was grams of dry weight BC produced 
per 1 liter fermentation volume. The BC yield reported in this study was mean \pm standard deviation from triplicate experiments.

TABLE 3: Primers Used in the Study to Identify the Microbes.

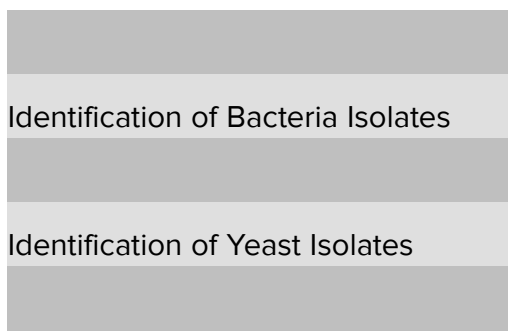

\begin{tabular}{|c|}
\hline Primers \\
\hline $27 F$ \\
\hline $1492 \mathrm{R}$ \\
\hline NL1 \\
\hline NL4
\end{tabular}

Sequence
5'-AGAGTTTGATCCTGGCTCAG-3'
5'-GGTTACCTTGTTACGACTT-3'
5/-GCATATCAATAAGCGGAGGAAAAG-3,
5/-GGTCCGTGTTTCAAGACGG-3,

\section{Result}

\subsection{Screening of Bacterial Cellulose-producing Isolates}

Bacteria which have been isolated and preserved from an adapted culture were chosen to be screened based on their visual colony difference. Prior to screening, 6 different colony types were subject to microscopy tests, showing that all bacteria were homogeneous in visual appearance, rod-shaped and pink when run through a gram staining procedure (Figure $\mathbf{3}$ ). This was an indication of acetic acid bacteria, common producer of $B C$.

After 14 days of static incubation, the single most productive bacteria, chosen through its exceptionally large thickness assessed through visual analysis, was selected for isolation. This isolate was the only bacterial inoculum used for the following experiments. This bacteria is stored in the i3L culture collection labeled "13.7.KBC.HSA".

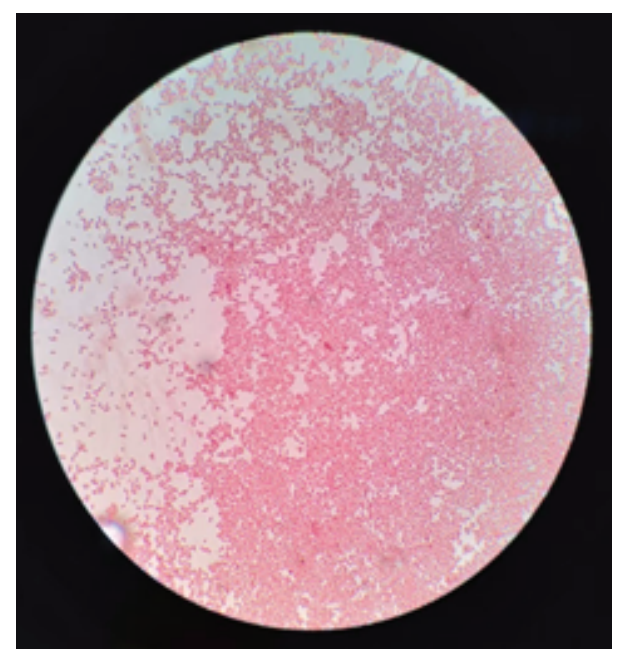

Figure 3: Visualization of gram stained bacteria under light microscope at 1000x magnification. 


\subsection{PCR analysis of Bacterial and Yeast Isolates}

Colony PCR was successful without any pretreatment of bacterial DNA template, where 4 out of 6 samples shows single bands (Figure 4). The remaining samples, "13.7.KBC.HSA" and the yeast needed Wizard ${ }^{\circledR}$ Genomic DNA Purification Kit prior to PCR efforts before having its NA validated for purity.

\subsection{Sequence Analysis}

PCR product would then be subjected to DNA sequencing. The resulting sequencing data were processed with FinchTV software to select clean peaks for both forward and reverse primers. The forward primer was then aligned with the reverse-complement of the reverse primer to validate the integrity of both the forward and reverse identity with the help of CLC Viewer software, resulting in a "Consensus" sequence (Appendix). This consensus sequence was then screened using nucleotide BLAST to provide microbial identity. The identity chosen from the BLAST result list would be based on the accession link's journal status, as either published or submitted. Unpublished BLAST results would be overlooked. 5 bacteria and 1 yeast have been successfully sequenced and identified (Table 4). All 5 of the bacteria are from closely related genera. Single isolated yeast belongs to the Brettanomyces genus.

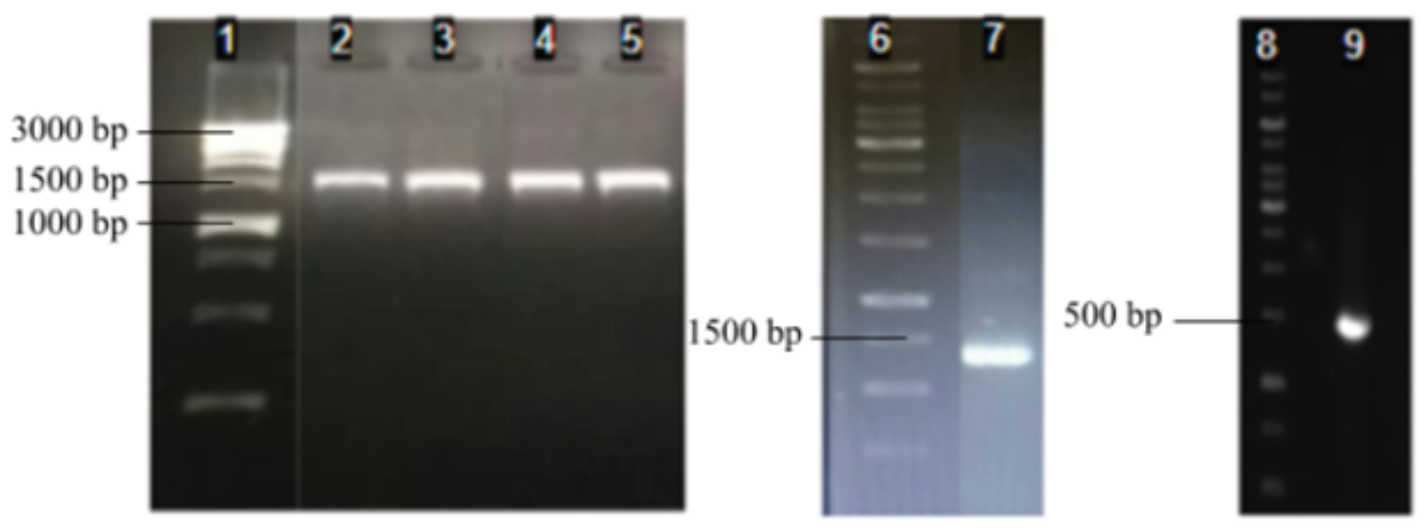

Figure 4: PCR gel electrophoresis showing 1. DNA Ladder $1 \mathrm{~kb}$ 2. "13.7.KBC.HSB" 3. "13.7.KBC.HSC" 4. "13.7.KBC.HSD" 5. "13.7.KBC.HSE" 6. DNA Ladder $1 \mathrm{~kb}$ 7. "13.7.KBC.HSA" 8. DNA Ladder 1 kb 9. "13.7.KBC.HSF".

\subsection{Co-Culture Fermentation Yields}

The resulting $\mathrm{BC}$ dry weight of the co-culture fermented for 14 days using molasses medium using $500 \mathrm{mg} / \mathrm{L}$ caffeine and $150 \mathrm{~g} / \mathrm{L}$ molasses prepared in acetate buffer (200 
TABLE 4: Isolated bacteria and Yeast Names, Primers, Identities and Their Accession.

\begin{tabular}{|c|c|c|c|c|c|}
\hline No. & Sample Name & $\begin{array}{l}\text { Forward } \\
\text { Primer }\end{array}$ & $\begin{array}{c}\text { Reverse } \\
\text { Primer }\end{array}$ & Identity & $\begin{array}{l}\text { Accession } \\
\text { Number }\end{array}$ \\
\hline 1 & 13.7.KBC.HSA & $27 \mathrm{~F}$ & $1492 R$ & $\begin{array}{l}\text { Komagataeibacter sp. } \\
\text { DS1MA.62A }\end{array}$ & LN884048.1 \\
\hline 2 & 13.7.KBC.HSB & $27 \mathrm{~F}$ & $1492 R$ & Komagataeibacter xylinus & MH511551.1 \\
\hline 3 & 13.7.KBC.HSC & $27 \mathrm{~F}$ & $1492 R$ & $\begin{array}{l}\text { Komagataeibacter } \\
\text { saccharivorans }\end{array}$ & KY287776.1 \\
\hline 4 & 13.7.KBC.HSD & $27 \mathrm{~F}$ & $1492 R$ & Komagataeibacter xylinus & MH511551.1 \\
\hline 5 & 13.7.KBC.HSE & $27 \mathrm{~F}$ & $1492 R$ & $\begin{array}{l}\text { Gluconacetobacter } \\
\text { saccharivorans }\end{array}$ & JF718412.1 \\
\hline 6 & 13.7.KBC.HSF & NL1 & NL4 & $\begin{array}{l}\text { Brettanomyces } \\
\text { bruxellensis }\end{array}$ & MH930867.1 \\
\hline
\end{tabular}

$\mathrm{mM}, \mathrm{pH} 4.75$ ) was calculated into an average yield of $27.7 \pm 1.83 \mathrm{~g} / \mathrm{L}$ of $\mathrm{BC}$. This data was expressed as mean \pm standard deviation from triplicate experiments.

\section{Discussion}

PCR analysis of the microbes were intended to identify the bacteria responsible for the monoculture setups as well as to highlight the diversity of the kombucha consortium. A total of 6 samples, despite some not as efficient as the other with producing BC, were sequenced. Furthermore, as only a single phenotype of yeast colony has emerged in all of the isolation efforts, following the usage of this yeast in the experimental coculture design, it could be of interest to investigate this particular yeast deeper. A single dominant yeast throughout every single time point in kombucha culture contradicts several studies which provide various species of yeast [9]. However, the prevalence of Brettanomyces in this experiment might be attributed to its affinity to the particular medium used in this experiment, as well as the lack of supportive medium for the uncultured yeast species.

Sequencing data has shown that the bacteria culture used in the co-culture experiments was identified as Komagataeibacter sp. DS1MA.62A. This BLAST result has been selected due to its journal status as submitted. Komagataeibacter is a genus of bacteria best known for its capability to produce cellulose. It is an acetic acid producing bacteria, gram-negative and aerobic. The particular species, Komagataeibacter sp. DS1MA.62A, has not been published in an article specifically for its BC producing capabilities. As for the yeast, it was identified as Brettanomyces bruxellensis, a yeast commonly used for 
brewing alcoholic beverages. This particular yeast have been isolated from a kombucha fermentation and have shown to be part of a greater consortium [9], unlike in this study where it is the sole isolate of yeast grown on PDA. This might also be attributed to dissimilarity of isolation methods used in the mentioned study.

The average yield of $\mathrm{BC}$ produced with a co-culture of Komagataeibacter $s p$. DS1MA.62A and Brettanomyces bruxellensis was $27.7 \mathrm{~g} / \mathrm{L}$ for 14 days. In comparison with previous study [17], a fermentation of 6 days using pineapple waste media with a pure Acetobacter xylinum 0416 culture yields $28.3 \mathrm{~g} / \mathrm{L}$ of BC. This sparse difference showed that the isolated culture had comparable potential with the known highest BC producing species.

\section{Conclusion}

This study isolated and screened the microbes in kombucha culture which were predominant after adaptation in molasses medium.. The result revealed 5 dominant bacteria and 1 dominant yeast. The bacterial isolates include Komagataeibacter sp. DS1MA.62A, Komagataeibacter xylinus, Komagataeibacter saccharivorans, Komagataeibacter xylinus and Gluconacetobacter saccharivorans. Meanwhile, the single isolated yeast was identified as Brettanomyces bruxellensis. Amongst the bacterial isolates, Komagataeibacter sp. DS1MA.62A was found to be the most potential BC producer, which has the ability to produce $B C$ at significant amount in monoculture fermentation. In co-culture fermentation between the selected bacteria and the yeast, Brettanomyces bruxellensis yeast was present in the culture to help break down complex sugar and producing ethanol to enhance the fermentation process. The BC yield from co-culture fermentation reached $27.7 \pm 1.83 \mathrm{~g} / \mathrm{L}$. This finding could be further used to consider which species of bacteria and yeast used during bacterial cellulose production in industrial scale which utilized molasses agroindustrial waste.

\section{Acknowledgment}

The authors are thankful to our project fellow, Jeffry and Khoe Erick Sudibya, who have worked tirelessly to aid, assist, and work alongside us inside and outside the laboratory throughout this study. We are grateful to do this project with you for the past half year. We would also like to thank i3L lab assistants who have helped us for the project. 


\title{
Funding
}

The study was funded by RISTEK DIKTI through PKM-GT (Program Kreativitas Mahasiswa-Gagasan Tertulis).

\section{Conflict of Interest}

None declared.

\section{APPENDICES}

\author{
13.7.KBC.HSA, Komagataeibacter sp. DS1MA.62A
}

CAGTCGCACGAACCTTTCGGGGTTAGTGGCGGACGGGTGAGTAACGCGTAGGGATCTATC CACGGGTGGGGGATAACTTTGGGAAACTGAAGCTAATACCGCATGACACCTGAGGGTCA AAGGCGCAAGTCGCCTGTGGAGGAACCTGCGTTCGATTAGCTAGTTGGTGGGGTAAAGG CCTACCAAGGCGATGATCGATAGCTGGTCTGAGAGGATGATCAGCCACACTGGGACTGA GACACGGCCCAGACTCCTACGGGAGGCAGCAGTGGGGAATATTGGACAATGGGCGCAAG CCTGATCCAGCAATGCCGCGTGTGTGAAGAAGGTTTTCGGATTGTAAAGCACTTTCAGCG GGGACGATGATGACGGTACCCGCAGAAGAAGCCCCGGCTAACTTCGTGCCAGCAGCCGC GGTAATACGAAGGGGGCAAGCGTTGCTCGGAATGACTGGGCGTAAAGGGCGCGTAGGCG GTTGACACAGTCAGATGTGAAATTCCCGGGCTTAACCTGGGGGCTGCATTTGATACGTGG CGACTAGAGTGTGAGAGAGGGTTGTGGAATTCCCAGTGTAGAGGTGAAATTCGTAGATAT TGGGAAGAACACCGGTGGCGAAGGCGGCAACCTGGCTCATGACTGACGCTGAGGCGCGA AAGCGTGGGGAGCAAACAGGATTAGATACCCTGGTAGTCCACGCTGTAAACGATGTGTG CTGGATGTTGGGTGACTTTGTCATTCAGTGTCGTAGTTAACGCGATAAGCACACCGCCTG GGGAGTACGGCCGCAAGGTTGAAACTCAAAGGAATTGACGGGGGCCCGCACAAGCGGTG GAGCATGTGGTTTAATTCGAAGCAACGCGCAGAACCTTACCAGGGCTTGACATGCGGAG GCCGTGTCCAGAGATGGGCATTTCTCGCAAGAGACCTCCAGCACAGGTGCTGCATGGCTG TCGTCAGCTCGTGTCGTGAGATGTTGGGTTAAGTCCCGCAACGAGCGCAACCCTCGCCTT TAGTTGCCATCACGTTTGGGTGGGCACTCTAAAGGAACTGCCGGTGACAAGCCGGAGGA AGGTGGGGATGACGTCAAGTCCTCATGGCCCTTATGTCCTGGGCTACACACGTGCTACAA TGGCGGTGACAGTGGGAAGCCAGGTGGTGACACCGAGCCGATCTCAAAAAGCCGTCTCA GTTCGGATTGCACTCTGCAACTCGAGTGCATGAAGGTGGAATCGCTAGTAATCGCGGATC AGCATGCCGCGGTGAATACGTTCCCGGGCCTTGTACACACCGCCCGTCACACCAT 


\subsection{KBC.HSB, Komagataeibacter xylinus}

GCAGTCGCACGAACCTTTCGGGGTTAGTGGCGGACGGGTGAGTAACGCGTAGGGATCTGT CCATGGGTGGGGGATAACTTTGGGAAACTGAAGCTAATACCGCATGACACCTGAGGGTC AAAGGCGCAAGTCGCCTGTGGAGGAACCTGCGTTCGATTAGCTAGTTGGTGGGGTAAAG GCCTACCAAGGCGATGATCGATAGCTGGTCTGAGAGGATGATCAGCCACACTGGGACTG AGACACGGCCCAGACTCCTACGGGAGGCAGCAGTGGGGAATATTGGACAATGGGCGCAA GCCTGATCCAGCAATGCCGCGTGTGTGAAGAAGGTTTTCGGATTGTAAAGCACTTTCAGC GGGGACGATGATGACGGTACCCGCAGAAGAAGCCCCGGCTAACTTCGTGCCAGCAGCCG CGGTAATACGAAGGGGGCAAGCGTTGCTCGGAATGACTGGGCGTAAAGGGCGCGTAGGC GGTTTTAACAGTCAGATGTGAAATTCCTGGGCTTAACCTGGGGGCTGCATTTGATACGTT GAGACTAGAGTGTGAGAGAGGGTTGTGGAATTCCCAGTGTAGAGGTGAAATTCGTAGAT ATTGGGAAGAACACCGGTGGCGAAGGCGGCAACCTGGCTCATTACTGACGCTGAGGCGC GAAAGCGTGGGGAGCAAACAGGATTAGATACCCTGGTAGTCCACGCTGTAAACGATGTG TGCTGGATGTTGGGTGACTTTGTCATTCAGTGTCGTAGTTAACGCGATAAGCACACCGCCT GGGGAGTACGGCCGCAAGGTTGAAACTCAAAGGAATTGACGGGGGCCCGCACAAGCGGT GGAGCATGTGGTTTAATTCGAAGCAACGCGCAGAACCTTACCAGGGCTTGACATGCGGA GGCCGTGTCCAGAGATGGGCATTTCTCGCAAGAGACCTCCAGCACAGGTGCTGCATGGCT GTCGTCAGCTCGTGTCGTGAGATGTTGGGTTAAGTCCCGCAACGAGCGCAACCCTCGCCT TTAGTTGCCATCACGTCTGGGTGGGCACTCTAGAGGAACTGCCGGTGACAAGCCGGAGGA AGGTGGGGATGACGTCAAGTCCTCATGGCCCTTATGTCCTGGGCTACACACGTGCTACAA TGGCGGTGACAGTGGGAAGCCAGGTAGCGATACCGAGCCGATCTCTAAAAGCCGTCTCA GTTCGGATTGCACTCTGCAACTCGAGTGCATGAAGGTGGAATCGCTAGTAATCGCGGATC AGCATGCCGCGGTGAATACGTTCCCGGGCCTTGTACACACCGCCCGTCACACCATGGGAG TTGGTTTG 


\subsection{KBC.HSC, Komagataeibacter saccharivorans}

CGCACGAACCTTTCGGGGTTAGTGGCGGACGGGTGAGTAACGCGTAGGGATCTGTCCATG GGTGGGGGATAACTTTGGGAAACTGAAGCTAATACCGCATGACACCTGAGGGTCAAAGG CGCAAGTCGCCTGTGGAGGAACCTGCGTTCGATTAGCTAGTTGGTGGGGTAAAGGCCTAC CAAGGCGATGATCGATAGCTGGTCTGAGAGGATGATCAGCCACACTGGGACTGAGACAC GGCCCAGACTCCTACGGGAGGCAGCAGTGGGGAATATTGGACAATGGGCGCAAGCCTGA TCCAGCAATGCCGCGTGTGTGAAGAAGGTTTTCGGATTGTAAAGCACTTTCAGCGGGGAC GATGATGACGGTACCCGCAGAAGAAGCCCCGGCTAACTTCGTGCCAGCAGCCGCGGTAA TACGAAGGGGGCAAGCGTTGCTCGGAATGACTGGGCGTAAAGGGCGCGTAGGCGGTTTT AACAGTCAGATGTGAAATTCCTGGGCTTAACCTGGGGGCTGCATTTGATACGTTGAGACT AGAGTGTGAGAGAGGGTTGTGGAATTCCCAGTGTAGAGGTGAAATTCGTAGATATTGGG AAGAACACCGGTGGCGAAGGCGGCAACCTGGCTCATTACTGACGCTGAGGCGCGAAAGC GTGGGGAGCAAACAGGATTAGATACCCTGGTAGTCCACGCTGTAAACGATGTGTGCTGG ATGTTGGGTGACTTTGTCATTCAGTGTCGTAGTTAACGCGATAAGCACACCGCCTGGGGA GTACGGCCGCAAGGTTGAAACTCAAAGGAATTGACGGGGGCCCGCACAAGCGGTGGAGC ATGTGGTTTAATTCGAAGCAACGCGCAGAACCTTACCAGGGCTTGACATGCGGAGGCCGT GTCCAGAGATGGGCATTTCTCGCAAGAGACCTCCAGCACAGGTGCTGCATGGCTGTCGTC AGCTCGTGTCGTGAGATGTTGGGTTAAGTCCCGCAACGAGCGCAACCCTCGCCTTTAGTT GCCATCACGTCTGGGTGGGCACTCTAGAGGAACTGCCGGTGACAAGCCGGAGGAAGGTG GGGATGACGTCAAGTCCTCATGGCCCTTATGTCCTGGGCTACACACGTGCTACAATGGCG GTGACAGTGGGAAGCCAGGTAGCGATACCGAGCCGATCTCTAAAAGCCGTCTCAGTTCG GATTGCACTCTGCAACTCGAGTGCATGAAGGTGGAATCGCTAGTAATCGCGGATCAGCAT GCCGCGGTGAATACGTTCCCGGGCCTTGTACACACCGCCCGTCACACCATGGGAGTTGGT TTGAC

\subsection{KBC.HSD, Komagataeibacter xylinus}

GCAGTCGCACGAACCTTTCGGGGTTAGTGGCGGACGGGTGAGTAACGCGTAGGGATCTGT CCATGGGTGGGGGATAACTTTGGGAAACTGAAGCTAATACCGCATGACACCTGAGGGTC AAAGGCGCAAGTCGCCTGTGGAGGAACCTGCGTTCGATTAGCTAGTTGGTGGGGTAAAG GCCTACCAAGGCGATGATCGATAGCTGGTCTGAGAGGATGATCAGCCACACTGGGACTG AGACACGGCCCAGACTCCTACGGGAGGCAGCAGTGGGGAATATTGGACAATGGGCGCAA GCCTGATCCAGCAATGCCGCGTGTGTGAAGAAGGTTTTCGGATTGTAAAGCACTTTCAGC GGGGACGATGATGACGGTACCCGCAGAAGAAGCCCCGGCTAACTTCGTGCCAGCAGCCG CGGTAATACGAAGGGGGCAAGCGTTGCTCGGAATGACTGGGCGTAAAGGGCGCGTAGGC GGTTTTAACAGTCAGATGTGAAATTCCTGGGCTTAACCTGGGGGCTGCATTTGATACGTT GAGACTAGAGTGTGAGAGAGGGTTGTGGAATTCCCAGTGTAGAGGTGAAATTCGTAGAT ATTGGGAAGAACACCGGTGGCGAAGGCGGCAACCTGGCTCATTACTGACGCTGAGGCGC GAAAGCGTGGGGAGCAAACAGGATTAGATACCCTGGTAGTCCACGCTGTAAACGATGTG TGCTGGATGTTGGGTGACTTTGTCATTCAGTGTCGTAGTTAACGCGATAAGCACACCGCCT GGGGAGTACGGCCGCAAGGTTGAAACTCAAAGGAATTGACGGGGGCCCGCACAAGCGGT GGAGCATGTGGTTTAATTCGAAGCAACGCGCAGAACCTTACCAGGGCTTGACATGCGGA GGCCGTGTCCAGAGATGGGCATTTCTCGCAAGAGACCTCCAGCACAGGTGCTGCATGGCT GTCGTCAGCTCGTGTCGTGAGATGTTGGGTTAAGTCCCGCAACGAGCGCAACCCTCGCCT TTAGTTGCCATCACGTCTGGGTGGGCACTCTAGAGGAACTGCCGGTGACAAGCCGGAGGA AGGTGGGGATGACGTCAAGTCCTCATGGCCCTTATGTCCTGGGCTACACACGTGCTACAA TGGCGGTGACAGTGGGAAGCCAGGTAGCGATACCGAGCCGATCTCTAAAAGCCGTCTCA GTTCGGATTGCACTCTGCAACTCGAGTGCATGAAGGTGGAATCGCTAGTAATCGCGGATC AGCATGCCGCGGTGAATACGTTCCCGGGCCTTGTACACACCGCCCGTCACACCATGGGAG TTGGTTTG 


\subsection{KBC.HSE, Gluconacetobacter saccharivorans}

CCTGATCCAGCAATGCCGCGTGTGTGAAGAAGGTTTTCGGATTGTAAAGCACTTTCAGCG GGGACGATGATGACGGTACCCGCAGAAGAAGCCCCGGCTAACTTCGTGCCAGCAGCCGC GGTAATACGAAGGGGGCAAGCGTTGCTCGGAATGACTGGGCGTAAAGGGCGCGTAGGCG GTTTTAACAGTCAGATGTGAAATTCCTGGGCTTAACCTGGGGGCTGCATTTGATACGTTG AGACTAGAGTGTGAGAGAGGGTTGTGGAATTCCCAGTGTAGAGGTGAAATTCGTAGATA TTGGGAAGAACACCGGTGGCGAAGGCGGCAACCTGGCTCATTACTGACGCTGAGGCGCG AAAGCGTGGGGAGCAAACAGGATTAGATACCCTGGTAGTCCACGCTGTAAACGATGTGT GCTGGATGTTGGGTGACTTTGTCATTCAGTGTCGTAGTTAACGCGATAAGCACACCGCCT GGGGAGTACGGCCGCAAGGTTGAAACTCAAAGGAATTGACGGGGGCCCGCACAAGCGGT GGAGCATGTGGTTTAATTCGAAGCAACGCGCAGAACCTTACCAGGGCTTGACATGCGGA GGCCGTGTCCAGAGATGGGCATTTCTCGCAAGAGACCTCCAGCACAGGTGCTGCATGGCT GTCGTCAGCTCGTGTCGTGAGATGTTGGGTTAAGTCCCGCAACGAGCGCAACCCTCGCCT TTAGTTGCCATCACGTCTGGGTGGGCACTCTAGAGGAACTGCCGGTGACAAGCCGGAGGA AGGTGGGGATGACGTCAAGTCCTCATGGCCCTTATGTCCTGGGCTACACACGTGCTACAA TGGCGGTGACAGTGGGAAGCCAGGTAGCGATACCGAGCCGATCTCTAAAAGCCGTCTCA GTTCGGATTGCACTCTGCAACTCGAGTGCATGAAGGTGGAATCGCTAGTAATCGCGGATC AGCATGCCGCGGTGAATACGTTCCCGGGCCTTGTACACACCGCCCGTCACACCATGGGAG TTGGTTTGACCTTAAGCCGGTGAGCGAACCG

\subsection{KBC.HSF, Brettanomyces bruxellensis}

GAAAAGAAACCAACAGGGATTGCCCCAGTAATGGCGAATGAAGCGGCAAGAGCCCAAAT TTGAAATCGGGCAACCGAGTTGTAATTTGGAGACGGGACACTAGAGAGGAGGAAGGCGA TTAAGTGCCTTGGAACAGGCTGCCGTAGAGGGTGAGAGCCCCGTGAATCGCTGGAGACC GATCAATTAGTGCCCGCCGAAGAGTCGAGTTGTTTGGGAATGCAGCTCTAAGTGGGTGGT ATATTCCATCTAAGGCTAAATATTAGCGAGAGACCGATAGCAAACAAGTACAGTGATGG AAAGATGAAAAGAACTTTGGAAAGAGAGTGAAATAGTACGTGAAATTGTTGAAAGGGAA GGGTATTTGATCCGACATGGTGTTTAGCAGCGGCCCGTTCCTCGTGGATGGGTGCACCTG GTTTACACTGGGCCAGCATCGGTTCTGGGAGCCATATACGGGGTTCGTGAATGTGGCCCT TCGATTCTGTCGGAGGGTGTTATAGCGCGGACATCTTGTGGCTAGCCGGGACCGGGGACT GCGGTGACTTGTCACCAAGGATGCTGGCAGAACGAGCAAATACCACCC

\section{References}

[1] Jayabalan, R., Malbaša, R. V., Lončar, E. S., Vitas, J. S., \& Sathishkumar, M. (2014). A review on kombucha tea-microbiology, composition, fermentation, beneficial effects, toxicity, and tea fungus. Comprehensive Reviews in Food Science and Food Safety, 13(4), 538--550. http://doi.org/10.1111/1541-4337.12073

[2] Chakravorty, S., Bhattacharya, S., Chatzinotas, A., Chakraborty, W., Bhattacharya, D., \& Gachhui, R. (2016). Kombucha tea fermentation: Microbial and biochemical dynamics. International Journal of Food Microbiology, 220, 63--72. 
[3] Chawla, P. R., Bajaj, I. B., Survase, S. A., \& Singhal, R. S. (2009). Bacterial cellulose: Fermentative production and applications. Food Technology and Biotechnology, 47(2), 107--124.

[4] Huang, Y., Zhu, C., Yang, J., Nie, Y., Chen, C., \& Sun, D. (2014). Recent advances in bacterial cellulose. Cellulose, 21(1), 1--30. http://doi.org/10.1007/s10570-013-0088-Z

[5] Lee, K. Y., Buldum, G., Mantalaris, A., \& Bismarck, A. (2014). More than meets the eye in bacterial cellulose: Biosynthesis, bioprocessing, and applications in advanced fiber composites. Macromolecular Bioscience, 14(1), 10--32. http://doi.org/10.1002/ mabi.201300298

[6] Cakar, F, Özer, I., Aytekin, A.Ö., \& Şahin, F. (2014). Improvement Production of Bacterial Cellulose by Semi-Continuous process in Molasses Medium. Carbohydrate Polymers 106, 7--13.

[7] Carreira, P., Mendes, J., A., Trovatti, E., Serafim, L., S., Freire, C., S., Silvestre, A., J., \& Neto, C., P. (2011). Utilization of residues from agro-forest industries in the production of high value bacterial cellulose. Bioresource Technol, 102(15):7354--7360.

[8] Hong, F., Zhu, Y., Yang, G., \& Yang, X. (2011). Wheat straw acid hydrolysate as a potential cost-effective feedstock for production of bacterial cellulose. J Chem Technol Biot, 86(5):675--680.

[9] Teoh, A. L., Heard, G., \& Cox, J. (2004). Yeast ecology of Kombucha fermentation. International Journal of Food Microbiology, 95(2), 119--126. http://doi.org/10.1016/j. ijfoodmicro.2003.12.020

[10] Liu, C., Hsu, W., Lee, F., \& Liao, C. (1996). The isolation and identification of microbes from a fermented tea beverage, Haipao, and their interactions during Haipao fermentation. Food Microbiology, 13(6), 407-415. doi:10.1006/fmic.1996.0047

[11] Naritomi, T., Kouda, T., Yano, H., \& Yoshinaga, F. (1998). Effect of ethanol on bacterial cellulose production from fructose in continuous culture. Journal of Fermentation and Bioengineering, 85(6), 598-603. doi:10.1016/s0922-338x(98)80012-3

[12] Krystynowicz, A., Czaja, W., Wiktorowska-Jezierska, A., Gonçalves-Miśkiewicz, M., Turkiewicz, M., \& Bielecki, S. (2002). Factors affecting the yield and properties of bacterial cellulose. Journal of Industrial Microbiology \& Biotechnology, 29(4), 189195. doi:10.1038/sj/jim/7000303

[13] Villarreal-Soto, S. A., Beaufort, S., Bouajila, J., Souchard, J., \& Taillandier, P. (2018). Understanding Kombucha Tea Fermentation: A Review. Journal of Food Science, 83(3), 580-588. doi:10.1111/1750-3841.14068 
[14] Marsh, A. J., Osullivan, O., Hill, C., Ross, R. P., \& Cotter, P. D. (2014). Sequence-based analysis of the bacterial and fungal compositions of multiple kombucha (tea fungus) samples. Food Microbiology, 38, 171-178. doi:10.1016/j.fm.2013.09.003

[15] Goh, W. N., Rosma, A., Kaur, B., Fazilah, A., Karim, A. A., \& Bhat, R. (2012). Fermentation of black tea broth (kombucha): I. effects of sucrose concentration and fermentation time on the yield of microbial cellulose. International Food Research Journal, 19(1), 109--117. http://doi.org/10.1016/j.pec.2010.07.039

[16] Coton, M., Pawtowski, A., Taminiau, B., Deniel, F., Coulloumme-labarthe, L., Fall, A., ... Coton, E. (2017). Unraveling microbial ecology of industrial-scale Kombucha fermentations by metabarcoding and culture-based methods. FEMS Microbiology Ecology, 95(3). https://doi.org/10.1093/femsec/fix048

[17] Zahan, K. A., Pa'e, N., \& Muhamad, I. I. (2015). Monitoring the Effect of pH on Bacterial Cellulose Production and Acetobacter xylinum 0416 Growth in a Rotary Discs Reactor. Arabian Journal for Science and Engineering, 40(7), 1881--1885. http://doi.org/10. 1007/s13369-015-1712-z 